\title{
Change of serum cytokine profiles by propranolol treatment in patients with infantile hemangioma
}

\author{
Sayaka Tani ${ }^{1}$, Kayo Kunimoto ${ }^{1, *}$, Yutaka Inaba ${ }^{1}$, Naoya Mikita ${ }^{1}$, Chikako Kaminaka ${ }^{1}$, \\ Nobuo Kanazawa ${ }^{1}$, Yuki Yamamoto ${ }^{1}$, Nobuyuki Kakimoto ${ }^{2}$, Tomohiro Suenaga ${ }^{2}$, \\ Takashi Takeuchi ${ }^{2}$ Hiroyuki Suzuki ${ }^{2}$, Masatoshi Jinnin ${ }^{1}$ \\ ${ }^{1}$ Department of Dermatology, Wakayama Medical University, Wakayama, Japan; \\ ${ }^{2}$ Department of Pediatrics, Wakayama Medical University, Wakayama, Japan.
}

SUMMARY Infantile hemangioma is a benign cutaneous tumor, which sometimes rapidly enlarges, causes cosmetic problem, destroys normal tissue, and possibly threatens life. Dye lasers, steroid administration, and watchful waiting had been the treatment options for infantile hemangioma, but in recent years propranolol therapy has become available. The mechanism underlying the action of propranolol, however, is still unknown. We hypothesized that cytokines whose expressions change before and during the treatment are responsible for the efficacy of the drug. This study aims to prove the hypothesis using patients' sera and membrane array. In this study, the serum cytokine concentrations of five patients with infantile hemangioma were measured using membrane array of 20 angiogenic cytokines. We compared them before and during propranolol treatment to identify the cytokines responsible for the effect of propranolol. Signals for angiogenin, epidermal growth factor (EGF), platelet-derived growth factor-BB (PDGF-BB), regulated on activation, normal T-cell expressed and secreted chemokine (RANTES), tissue inhibitor of metalloproteinases 1 (TIMP-1), and tissue inhibitor of metalloproteinases 2 (TIMP-2) were evident in all five cases before treatment. Furthermore, PDGFBB was the only cytokine of which concentration was decreased during treatment with statistically significant difference. This report is a pilot study with a small number of samples, and further detailed research with increased number of samples is necessary. Nonetheless, our results suggest that PDGFBB may be involved in the action of propranolol. In addition, its serum concentration can be utilized as a potential marker of the therapeutic effect.

Keywords infantile hemangioma, propranolol, PDGF-BB, cytokine, angiogenesis

\section{Introduction}

Infantile hemangioma is a benign cutaneous tumor caused by proliferation of vascular endothelial cells. The lesions typically appear on the head or face around 1-2 weeks after birth. They tend to grow until one year (proliferating phase), after which they start to involute (involution phase) (1). Larger lesions, however, may cause organ failures (e.g. visual impairment, eating disorder, airway obstruction, or heart failure), and sometimes even threaten life. They also become cosmetically problematic, leaving capillary dilation, skin relaxation, skin atrophy, and scarring.

Dye lasers, steroid administration, or watchful waiting had been the most common treatment options for infantile hemangioma (2). Since propranolol recently became available, its dramatic effects have been reported. However, the mechanism underlying its action is still unknown. We hypothesized that cytokines whose expressions change before and during the treatment are responsible for the efficacy of the drug. This study aims to prove the hypothesis using patients' sera and membrane array.

\section{Materials and Methods}

\subsection{Clinical assessment and patient material}

Serum samples were obtained from five patients with infantile hemangioma (Table 1) before and during propranolol treatment. The age of the patients at the first visit was between three and six months. Three cases had hemangiomas on the head or face, and one case had lesion on the trunk. Case 2 had lesions on both the face and trunk. The clinical subtype according to the depth of soft tissue involvement is as follows: Two cases of 
Table 1. Clinical features of infantile hemangioma cases in this study

\begin{tabular}{|c|c|c|c|c|c|c|}
\hline Case & age (months) & $\operatorname{sex}$ & site & type & period of treatment (months) & Dose of propranolol \\
\hline 1 & 3 & M & cheek & mixed & 3 & $3 \mathrm{mg} / \mathrm{kg} /$ day \\
\hline 2 & 3 & M & $\begin{array}{l}\text { forehead } \\
\text { abdomen }\end{array}$ & $\begin{array}{l}\text { mixed } \\
\text { mixed }\end{array}$ & 5 & $3 \mathrm{mg} / \mathrm{kg} /$ day \\
\hline 3 & 6 & $\mathrm{~F}$ & head & superficial & 4 & $3 \mathrm{mg} / \mathrm{kg} /$ day \\
\hline 4 & 4 & $\mathrm{~F}$ & buttock & superficial & 3 & $3 \mathrm{mg} / \mathrm{kg} /$ day \\
\hline 5 & 4 & M & forehead & deep & 4 & $3 \mathrm{mg} / \mathrm{kg} /$ day \\
\hline
\end{tabular}

superficial type, one case of deep type, and two cases of mixed type.

Propranolol administration was initiated at a dosage of $1 \mathrm{mg} / \mathrm{kg} /$ day orally divided twice daily, and then increased to $3 \mathrm{mg} / \mathrm{kg} /$ day in all cases. Cardiac screenings, such as chest X-ray, electrocardiogram, and 2-dimension echocardiogram, were performed before and during propranolol treatment. The serum samples were obtained within three hours after morning intake of propranolol. Propranolol successfully reduced the coloration and/or size of tumors in all cases at the time of serum sampling (3-5 month after the initiation, Table 1).

This study was approved by the Research Ethics Committee of Wakayama Medical University (No. 2730). Informed consent was obtained according to the Declaration of Helsinki.

\subsection{Measurement of serum cytokine levels}

Serum levels of 20 cytokines (angiogenin, epidermal growth factor (EGF), epithelial neutrophil activating peptide-78 (ENA-78), basic fibroblast growth factor (bFGF), growth-related oncogene (GRO), interferon- $\gamma$ (IFN- $\gamma$ ), insulin-like growth factors-1 (IGF-I), interleukin-6 (IL-6), interleukin-8 (IL-8), leptin, monocyte chemotactic protein 1 (MCP-1), plateletderived growth factor-BB (PDGF-BB), placental growth factor (PIGF), regulated on activation, normal T-cell expressed and secreted chemokine (RANTES), transforming growth factor- $\beta 1$ (TGF- $\beta 1$ ), tissue inhibitor of metalloproteinases 1 (TIMP-1), tissue inhibitor of metalloproteinases 2 (TIMP-2), thrombopoietin, vascular endothelial growth factor (VEGF), and VEGF-D) were measured using human angiogenesis antibody ArrayMembrane (Abcam, Cambridge, UK) (3). Biotinconjugated monoclonal antibody of each cytokine was precoated on the membranes.

As reported previously (3), an aliquot of serum was reacted with the antibodies on each membrane. The membrane was then incubated with labeled streptavidin, and detection buffer was added. Images were obtained using WSE-6,100 Lumino Graph (ATTO, Tokyo, Japan).

\subsection{Statistical analysis}

Statistical analysis was carried out with Mann-Whitney's $\mathrm{U}$ test for the comparison of medians. $p$ values $<0.05$ were considered significant.

\section{Results and Discussion}

3.1. Clinical features of infantile hemangioma patients in this study

In this study, propranolol was administered to various types of patients with infantile hemangioma (Table 1). The clinical images of all patients are shown in Figure 1. Hemangiomas were present on a three-month-old boy's cheek (mixed type), a three-month-old boy's forehead and abdomen (mixed type), a six-month-old girl's head (superficial type), a four-month-old girl's buttock (superficial type), and a four-month-old boy's forehead (deep type). Propranolol successfully reduced the coloration and/or size of tumors in all cases. No cardiac abnormalities were detected in all five cases before and during propranolol treatment.

\subsection{Serum cytokine expression profiles of infantile hemangioma}

Cytokine expression profiles in sera of patients with infantile hemangioma were analyzed using commercially available membrane array kits. As shown in Figure 2A, biotin-conjugated monoclonal antibodies of 20 cytokine (angiogenin, EGF, ENA-78, bFGF, GRO, IFN- $\gamma$, IGF-I, IL-6, IL-8, Leptin, MCP-1, PDGF-BB, PIGF, RANTES, TGF- $\beta 1$, TIMP-1, TIMP-2, thrombopoietin, VEGF, and VEGF-D) were precoated on the membrane. Aliquots of serum were added to the membrane, and then allowed to react with the antibodies at room temperature, after which signals were developed by labeled streptavidin. The signal densities of each antigen-specific antibody spot were obtained using 2-D densitometry and then quantified.

Signals for angiogenin, EGF, PDGF-BB, RANTES, TIMP-1, and TIMP-2 were evident in all five cases before treatment, whereas ENA-78, bFGF, GRO, IFN- $\gamma$, IGF-I, IL-6, IL-8, PIGF, TGF- $\beta 1$, thrombopoietin, VEGF, or VEGF-D were absent in all cases (Figure 2B). Fluctuations in expression were observed in the levels of Leptin and MCP-1.

Figure 3 shows percentage changes of cytokine expression during treatment compared with the values before treatment: Changes in the expression of eight cytokines were shown, excluding those that were not expressed both before and during treatment. The levels of EGF, RANTES, TIMP-1, and TIMP-2 tended to 


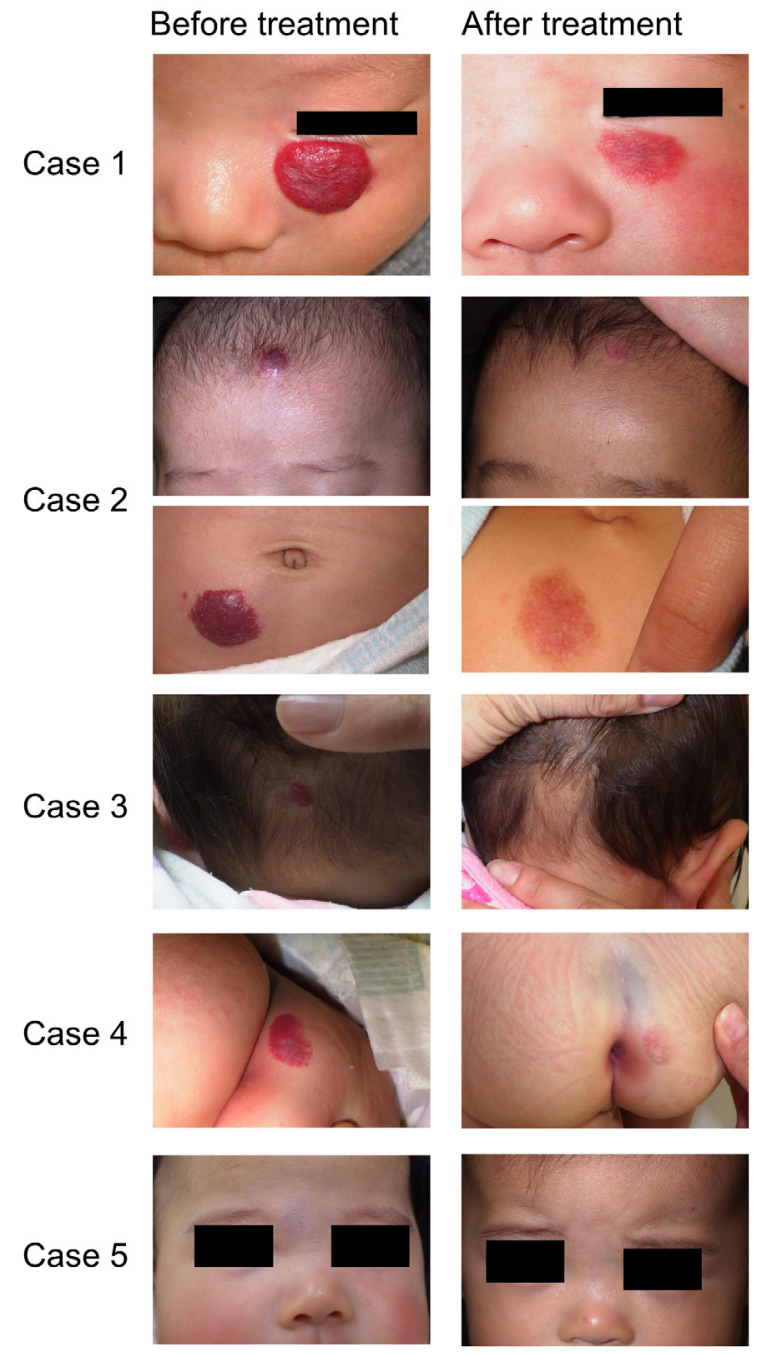

Figure 1. Clinical images of patient with infantile hemangioma included in this study. (left) Cases 1-5 before the treatment with propranolol, (right) Cases 1-5 during the propranolol treatment.

be increased during the treatment, albeit statistically insignificant. We found significant difference in the decrease of PDGF-BB levels during the treatment (Figure 3, $p<0.05$ ).

Various cytokines may participate in the tumorigenesis of infantile hemangioma. The involvement of VEGF, angiopoietin (4), TGF- $\beta$ (5), TNF- $\alpha$, and IL-1 (6) have already been described. Taran et al. also found that significantly higher TIMP-2 levels were observed in infantile hemangioma tissues at the mRNA level (7). Furthermore, serum cytokine levels have also been evaluated for their potential as tumor markers in this disease. For example, serum levels of VEGF were increased in infantile hemangioma at the proliferative or involution phase. In addition, serum MCP-1 and macrophage inflammatory protein-1 $\beta$ (MIP-1 $1 \beta$ ) may be utilized as the marker of regression (8). Similarly, propranolol treatment for proliferating infantile hemangioma can reduce the peripheral serum and urinary concentrations of epidermal growth factor-like protein 7 (EGFL7) levels (9).
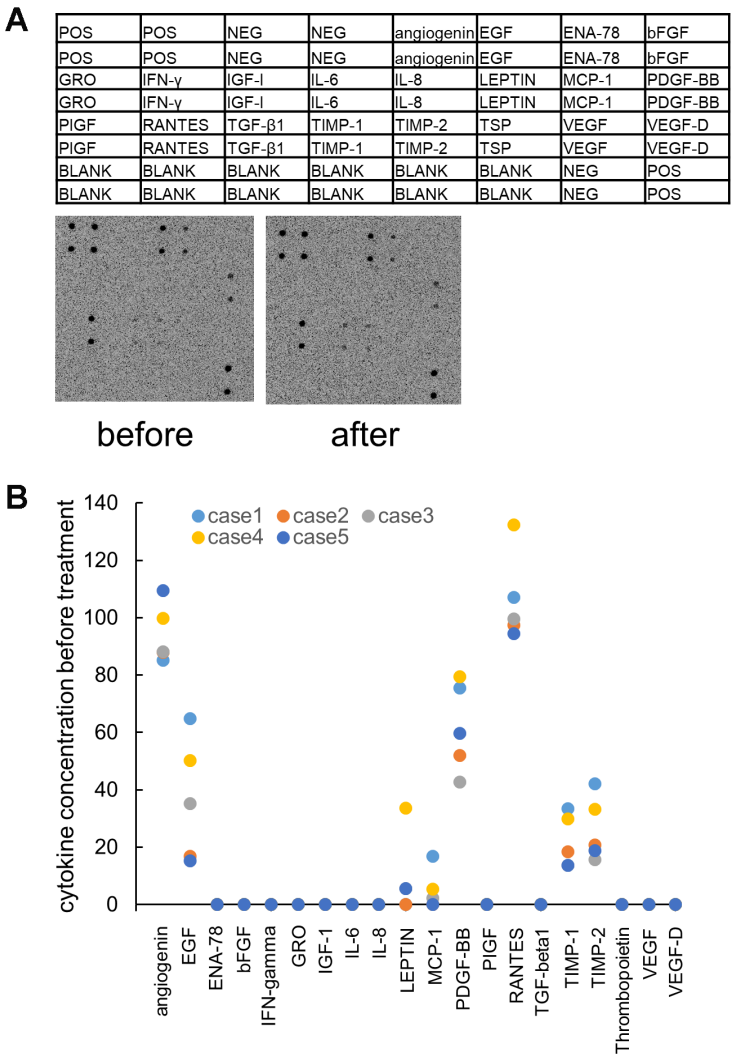

Figure 2. (A) Results of human angiogenesis antibody array. (upper panel) Array maps used in this study, (lower panel) Set of representative membranes before and during the propranolol treatment. (B) Cytokine concentration before the treatment. The relative expression levels of twenty cytokines measured by the array using sera of five patients with hemangioma before the treatment are shown on the ordinate. The density of positive control in each membrane was set at 100 .

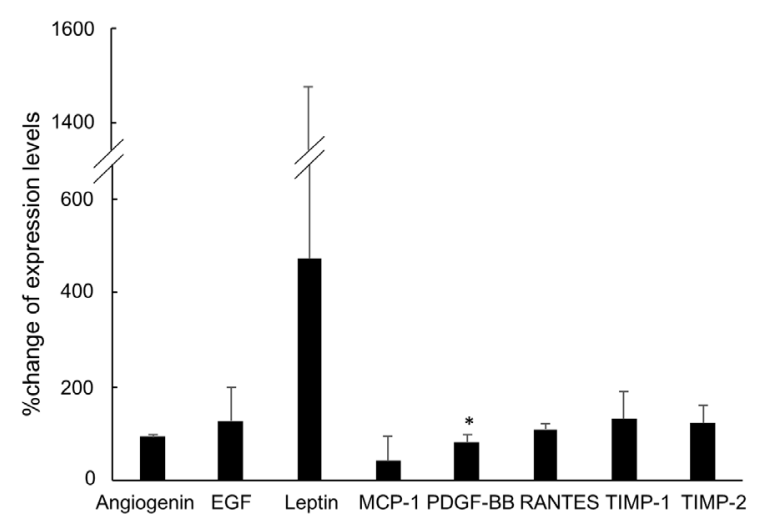

Figure 3. Change of cytokine expression levels measured by the array. The y-axis indicates the change of each cytokine level before and during the propranolol treatment (\%). Levels of cytokines unexpressed both before and during the treatment was not show. The bar graphs represent the mean values, and the error bars indicate standard deviations. " $p<0.05$. When signals were not detected, cytokine level was set at 1 for the statistical analysis.

In this study, we aimed to determine multiple serum cytokines at the same time using array experiments before and during administration of propranolol. Before the treatment, signals for angiogenin, EGF, PDGF- 
BB, RANTES, TIMP-1, and TIMP-2 were evident in all five cases. The levels of Leptin and MCP-1 were not consistent among five patients. The other cytokines were not expressed in all cases. Consistently, Jiang et al. reported that angiogenin levels are increased in the sera of proliferative hemangioma (10), and suggested them as the biomarker. Yamashita et al. also described that serum RANTES levels were significantly down-regulated in patients with progressive infantile hemangioma than those in non-progressive hemangioma group (3).

The second novel finding is that PDGF-BB was the only cytokine whose concentration was changed during treatment with statistically significant difference. PDGF is an angiogenic factor produced by various cell types including monocytes and macrophages, and is known to promote endothelial proliferation. Roach et al. reported that PDGF-BB inhibits differentiation into adipocytes in infantile hemangiomas and suggested the cytokine as a negative regulator of hemangioma regression (11). On the other hand, no relationship between propranolol and PDGF-BB has yet been reported.

Taken together, our results suggested two possibilities: (1) Propranolol suppresses PDGF-BB production from monocytes and macrophages, which promotes regression of hemangioma. (2) The total amount of PDGF produced from decreased number of tumor cells was reduced due to administration of propranolol. Considering the first possibility, PDGFBB may be involved in the action of propranolol. Furthermore, its serum concentration can be utilized as a marker of therapeutic effect. This report is a pilot study with a small number of samples, and there is limitation: In babies, growth may dramatically affect serum cytokine levels. However, control group without hemangioma for comparing the baseline levels and the subsequent cytokine changes as babies grow up were not included in this study. Further detailed research with larger number of samples and control patients (e.g. patients without hemangioma or propranolol administration) is necessary.

\section{References}

1. Boye E, Jinnin M, Olsen BR. Infantile hemangioma: Challenges, new insights, and therapeutic promise. J Craniofac Surg. 2009; 20:678-684.

2. Jinnin M, Ishihara T, Boye E, Oslen BR. Recent progress in studies of infantile hemangioma. J Dermatol. 2010; 37:283-298.

3. Yamashita T, Jinnin M, Makino K, Kajihara I, Aoi J, Masuguchi S, Fukushima S, Ihn H. Serum cytokine profiles are altered in patients with progressive infantile hemangioma. Biosci Trends. 2018; 12:438-441.

4. Yu Y, Varughese J, Brown LF, Muliken JB, Bischoff J. Increased Tie2 expression, enhanced response to angiopoietin-1, and dysregulated angiopoietin-2 expression in hemangioma-derived endothelial cells. Am J Pathol. 2001; 159:2271-2280.

5. Meng X, Deng CS, Wang QX, Wang TX, Liu WX. Expression and significance of pericytes and TGF-beta in infantile parotid hemangioma. Shanghai Kou Qiang Yi Xue. 2012; 21:687-690. (in Chinese).

6. Wu KQ, Muratore CS, So EY, Sun C, Dubielecka PM, Reginato AM, Liang OD. M1 macrophage-Induced endothelial-to-mesenchymal transition promotes infantile hemangioma regression. Am J Pathol. 2017; 187:21022111.

7. Taran K, Wnęk A, Kobos J, Andrzejewska E, Przewratil P. Tissue and serum mRNA profile of MMPs-2/9 as a potential novel biomarker for the most individual approach in infantile hemangiomas and cancer disease. Immunobiology. 2017; 222:1035-1042.

8. D'Arcangelo D, Nicodemi EM, Rossi S, Giampietri C, Facchiano F, Facchiano A. Identification of serum regression signs in infantile hemangioma. PLoS One. 2014; 9:e88545.

9. Bin L, Manli C, Jie L, Xiaopeng Y, Zhaoquan L, Zhongcheng G. Expression of serum and urinary vascular endothelial growth factor-A and epidermal growth factorlike domain 7 in proliferating hemangioma treated with propranolol. Hua Xi Kou Qiang Yi Xue Za Zhi. 2014; 32:441-445. (in Chinese).

10. Jiang $\mathrm{C}$, Lin $\mathrm{X}, \mathrm{Hu} \mathrm{X}$, Chen $\mathrm{H}$, Jin $\mathrm{Y}, \mathrm{Ma} \mathrm{G}$, Chen $\mathrm{D}$, Chen $\mathrm{X}, \mathrm{Gu} \mathrm{W}$. Angiogenin: A potential serum marker of infantile hemangioma revealed by cDNA microarray analysis. Plast Reconstr Surg. 2014; 134:231e-239e.

11. Roach EE, Chakrabarti R, Park NI, Keats EC, Yip J, Chan NG, Khan ZA. Intrinsic regulation of hemangioma involution by platelet-derived growth factor. Cell Death Dis. 2012; 3:e328.

Received April 7, 2020; Revised April 20, 2020; Accepted April 27, 2020

*Address correspondence to:

Dr. Kayo Kunimoto, Department of Dermatology, Wakayama Medical University Graduate School of Medicine, 811-1 Kimiidera, Wakayama, Wakayama, 641-0012, Japan.

E-mail:k-jigen@wakayama-med.ac.jp 